\title{
Subtherapeutic levels of voriconazole in continuous renal replacement therapy patients
}

\begin{abstract}
Voriconazole is an antifungal widely used in the treatment of invasive fungal infections, thus pharmacokinetic monitoring is highly important to achieve appropriate dosages. It has been described that patients undergoing continuous renal replacement therapy did not see plasma voriconazole concentrations altered. Two cases are presented in which subtherapeutic voriconazole plasma levels are obtained while the patients are in continuous venovenous hemodiafiltration.
\end{abstract}

Volume 8 Issue 2 - 2019

\author{
Manuel Azócar,' Marlon Barraza,' JP Torres, ${ }^{2}$ \\ Carlos Acuña, ${ }^{3}$ Jorge Morales, ${ }^{1}$ \\ 'Pharmaceutical Services, Hospital Dr. Luis Calvo Mackenna, \\ Chile \\ 2Departamento de Pediatria, Universidad de Chile, Chile \\ ${ }^{3}$ Intensive Care Unit, Hospital Dr. Luis Calvo Mackenna, Chile
}

Correspondence: Manuel Azócar, Pharmaceutical Services.
Hospital Dr. Luis Calvo Mackenna, Santiago, Chile,
Email jmorales@calvomackenna.cl

Received: November 19,2018 | Published: March 29, 2019

\section{Introduction}

Voriconazole is a broad-spectrum azole antifungal agent frequently used in the treatment of invasive fungal infections (IFI) generated by Aspergillus and other types of fungi. ${ }^{1}$ Due to the high inter and intrapatient variability, the monitoring of plasma concentrations (PC) is fundamental in the management of patients with IFI because PC less than $1.0 \mathrm{mcg} / \mathrm{mL}$ are associated with therapeutic failure. ${ }^{2,3}$ It has been described that the use of renal replacement therapies can alter pharmacokinetic parameters of various drugs in critically ill patients, which would generate under or overdose risks. ${ }^{4}$ Continuous venovenous hemodiafiltration (CVVHDF) stands out for its high efficiency in the removal of solutes and fluids, in addition to being applicable to patients with unstable hemodynamics. ${ }^{5}$ It has been described that voriconazol dose adjustment is not necessary in patients undergoing CVVHDF, and no change in PC was observed. ${ }^{4,6} \mathrm{We}$ identified two cases of patients who had low levels of voriconazole while receiving CVVHDF therapy.

\section{Case I}

An 8-years-old male patient, ALL 2CR, UCBT +63 , was admitted to the PICU from BMT Unit for respiratory decompensation secondary to cytomegalovirus pneumonia in treatment with ganciclovir, needing connection to IVM. The patient progressed favorably after 2 weeks, being able to do a ventilatory weaning. However, by the third week of treatment, the patient presented with new respiratory failure, which required reconnection to IVM and HFOV, so a meropenem and vancomycin treatment was added, considering the previous use of broad spectrum antibiotics. In addition, the child had renal failure that required CVVHDF. The patient developed persistent fever and elevated CRP after 4 days of antibiotic therapy. An IFI study was carried out and therapy with voriconazole $200 \mathrm{mg} / 12 \mathrm{~h}$ IV was initiated. After 5 days, levels were found to be at $0.20 \mathrm{mcg} / \mathrm{mL}$, so the dose was increased to $200 \mathrm{mg} / 8 \mathrm{~h}$. Later in new monitoring, a PC of $0.10 \mathrm{mcg} / \mathrm{mL}$ was discovered.

\section{Case 2}

A 9-year-old female patient, AML-M3 in recent relapse, was admitted to the PICU from the Oncology Unit due to hemodynamic and respiratory decompensation during myelogram, requiring support with vasoactive drugs and IMV. The patient advanced rapidly with renal failure, needing CVVHDF. The piperacillin/tazobactam and cloxacillin regimen was initiated, with no response after 3 days of treatment. The girl presented pulmonary images with inflammatory involvement and multiple nodules. Amphotericin B was started and BAL was performed with galactomannan in 0.93 and in blood 0.17 . Due to the severity of the patient, antifungal treatment was maintained. The patient persisted with fever, increased CRP, and an increased need for vasoactive drugs, escalating to meropenem and linezolid. Subsequently, due to persistence of fever and elevated CRP, new BAL was made and Aspergillus positive PCR was detected, so voriconazole $200 \mathrm{mg} / 12 \mathrm{~h} \mathrm{IV}$ was added. Levels were measured to be $0.10 \mathrm{mcg} / \mathrm{mL}$, so the dose was increased to $200 \mathrm{mg} / 8 \mathrm{~h}$ and then levels were again monitored, resulting in $\mathrm{PC}$ at $0.10 \mathrm{mcg} / \mathrm{mL}$. In case of suspicion of elimination by CVVHDF system, voriconazole levels were measured in ultrafiltrate, obtaining a value of $0.42 \mathrm{mcg} / \mathrm{mL}$.

\section{Discussion}

The studies of Veltri et al and Fuhrmann et al., show that it is not necessary to adjust dosages in patients undergoing CVVHDF, because the system would not alter PC. However, both patients had very low subtherapeutic $\mathrm{CP}$ while receiving HDFVVC, despite being treated with high doses of voriconazole, $20 \mathrm{mg} / \mathrm{kg} /$ day in case 1 and $14 \mathrm{mg} / \mathrm{kg} /$ day in case 2 . The elimination of drugs by renal replacement systems depends on physicochemical factors such as molecular size, plasma protein binding (PPB), solubility, volume of distribution, among others. Other relevant influences include factors of the system such as filter surface, pore size, extraction mechanisms and work modality used. ${ }^{6}$ Voriconazole has a molecular mass of 349Da, PPB 58\%, intermediate lipophilicity and wide distribution to tissues. Therefore, it should theoretically have a low elimination by renal replacement therapy. ${ }^{4,5}$ In these patients a high elimination was found, which may be due to some pharmacokinetic differences characteristic of pediatrics, considering that pharmacokinetic studies are performed in adult patients. One of the potentially highly variable interpatient factors is the free drug fraction dependent on plasma protein binding, however, none of the patients presented considerable hypoproteinemia. In case 1: albumin $3.0-3.1 \mathrm{~g} / \mathrm{dL}$ and total proteins $7.0-7.5 \mathrm{~g} / \mathrm{dL}$, in case 2 : albumin $3.4-3.9 \mathrm{~g} / \mathrm{dL}$ and total proteins $6.9-$ $7.4 \mathrm{~g} / \mathrm{dL}$. Thus, we cannot justify this phenomenon with this reason, also considering that voriconazole does not have high PPB. 
Considering drug-drug interactions that could alter voriconazole $\mathrm{PC}$, none of these patients received enzyme inducers before or during treatment with voriconazole. The first patient, 1 month before admission to PICU, was 1 week into treatment with voriconazole for a suspicion of invasive fungal infection, using $200 \mathrm{mg} / 8 \mathrm{~h} \mathrm{IV}$ obtained PC $1.79 \mathrm{mcg} / \mathrm{mL}$, not being at that time in renal replacement therapy. This suggests that it is not a phenomenon inherent in the physiology of the child. Finally, considering that voriconazole was found in considerable concentrations in the ultrafiltrate of the patient in case 2 , it can be said that there was a drug removal through the CVVHDF system.

\section{Conclusion}

The monitoring of plasma levels of voriconazole is essential in patients undergoing CVVHDF, and there may be individual phenomena that require different treatment schemes. More studies are needed to analyze the behavior of this drug in pediatric patients undergoing CVVHDF.

\section{Acknowledgments}

None.

\section{Conflicts of interest}

The author declares that there is no conflicts of interest.

\section{References}

1. Scott L, Simpson D. Voriconazole: a review of its use in the management of invasive fungal infections. Drugs. 2007;67(2):269-298.

2. Walsh T, Timothy Driscoll, Peter A Milligan, et al. Pharmacokinetics, safety, and tolerability of voriconazole in immunocompromised children. Antimicrob Agents Chemother. 2010;54(10):4116-4123.

3. Groll A, Tragiannidis A. Update on antifungal agents for paediatric patients. Clin Microbiol Infect. 2010;16(9):1343-1353.

4. Veltri MA, Neu AM, Fivush BA, et al. Drug dosing during intermittent hemodialysis and continuous renal replacement therapy : special considerations in pediatric patients. Paediatr Drugs. 2004;6(1):45-65.

5. Ricci Z, Goldstein SL. Pediatric Continuous Renal Replacement Therapy. Contrib Nephrol. 2016;187:121-130.

6. Fuhrmann V, Schenk P, Jaeger W, et al. Pharmacokinetics of voriconazole during continuous venovenous haemodiafiltration. $J$ Antimicrob Chemother. 2007;60(5):1085-1090. 